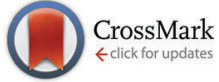

Cite this: Phys. Chem. Chem. Phys., 2015, 17, 1957

Received 17th August 2014 Accepted 21st November 2014

DOI: $10.1039 / \mathrm{c} 4 \mathrm{cp} 03683 \mathrm{~b}$

www.rsc.org/pccp

\title{
A facile synthesis of highly stable and luminescent Ag clusters: a steady-state and time-resolved spectroscopy study
}

\begin{abstract}
Nabin Kumar Pal and Carola Kryschi*
In this paper, we reported a very simple and environmentally friendly procedure for the synthesis of bright luminescent and nearly monodisperse Ag nanoclusters stabilized by a poly( $N$-vinylpyrrolidone) homopolymer. In this synthesis route acetonitrile or $\mathrm{N}, \mathrm{N}$-dimethylformamide (DMF) acts as both solvent and a reducing agent at their respective reflux temperatures. The as-prepared Ag clusters were found to be highly stable in various solvents as well as show nearly no changes in their emission intensity in solutions with different $\mathrm{pH}$ values and ionic strengths. Remarkably, the acetonitrile method predominantly produces blue emitting Ag clusters with a photoluminescence (PL) emission maximum at $424 \mathrm{~nm}$ (quantum yield 3.5\%), whereas mainly blue-green emitting Ag clusters with the PL emission maximum at $450 \mathrm{~nm}$ (quantum yield 2.7\%) were formed using the DMF method. The photo-physical, electronic, structural and morphological properties of the Ag clusters were investigated by performing UV/Vis absorption spectroscopy, stationary and time-resolved PL spectroscopy, $\mathrm{X}$-ray photoelectron spectroscopy, femtosecond transient absorption spectroscopy, and transmission electron microscopy experiments.
\end{abstract}

\section{Introduction}

Luminescent noble metal clusters are some of the most promising materials in nanoscience nowadays. ${ }^{1-8}$ Clusters having a size of less than $2 \mathrm{~nm}$ behave like a bridging entity between atoms and bulk materials. Due to such ultrasmall sizes being on the scale of the Fermi wavelength of the conduction-band electrons ( $c a .0 .7 \mathrm{~nm}$ ) and thereby, in the quantum confinement regime, ${ }^{9,10}$ noble metal clusters exhibit molecular-like electronic transitions between discrete energy levels and show new size-tunable optical and electronic properties. ${ }^{11-13}$ Sizetunable properties, along with high photostability and low cytotoxicity, make these clusters attractive candidates for versatile applications such as optical sensing, ${ }^{14-18}$ catalysis, ${ }^{19}$ bio-labelling, ${ }^{20,21}$ bio-imaging, ${ }^{22}$ single-particle optoelectronics, ${ }^{9}$ surface enhanced Raman spectroscopy ${ }^{23}$ and others.

Among the noble-metal clusters gold clusters have been extensively studied over the past two decades. However, only a few synthesis techniques for the preparation of stable luminescent silver (Ag) clusters are known. In recent past, the research activities on $\mathrm{Ag}$ clusters have enormously increased and since then, various synthesis procedures have been developed to produce stable luminescent $\mathrm{Ag}$ clusters that are stabilized by a variety of protecting ligands. Among various methods, template-based synthesis techniques

Department of Chemistry and Pharmacy and ICMM, Friedrich-Alexander University of Erlangen-Nuremberg, Erlangen, Germany.E-mail: carola.kryschi@fau.de are the most recognized ones. Different kinds of templates such as polymers, ${ }^{19,24,25}$ proteins, ${ }^{26,27}$ DNA, ${ }^{21,28}$ oligonucleotides, ${ }^{29,30}$ polyelectrolytes, ${ }^{31}$ dendrimers, ${ }^{32,33}$ and polymer microgels ${ }^{34}$ have been used so far. Small molecules, especially those containing carboxylic or thiol groups, ${ }^{35,36}$ were also utilized as stabilizers. Dickson and co-workers were the first to produce small Ag clusters by irradiating UV light onto a silver-oxide film. ${ }^{37}$ Later on, the same group reported highly stable and luminescent $\mathrm{Ag}$ and $\mathrm{Au}$ clusters incorporated into OH-terminated poly(amidoamine) (PAMAM) dendrimers in aqueous solution. ${ }^{32,33}$ Martinez et al. ${ }^{21,38}$ synthesized DNA-templated stable Ag clusters that were successfully applied for protein detection. Another novel synthesis procedure for bio-compatible, stable luminescent $\mathrm{Ag}$ and $\mathrm{Au}$ clusters was developed by Nienhaus et al. ${ }^{39}$ Recently Parak et $a .^{40}$ reported a synthesis route that enabled the preparation of water-soluble, red-luminescent $\mathrm{Ag}$ clusters with a mean size of $2.2 \pm 0.4 \mathrm{~nm}$. In this sophisticated synthesis procedure didodecyldimethylammonium stabilized Ag nanoparticles with sizes of around $4 \mathrm{~nm}$ were initially formed, which were subsequently size-reduced by a ligand exchange process followed by heating and UV light exposure. Other routes such as radiolytic, ${ }^{41}$ microwave irradiation, ${ }^{42}$ sonochemical, ${ }^{43}$ electrochemical ${ }^{44}$ methods have also been proven to be useful to prepare noble-metal clusters.

However most of the methods mentioned above require photo-irradiation, electric field application or an external reducing agent such as sodium borohydride or ascorbic acid. In recent past Liz-Marzán and co-workers ${ }^{45,46}$ could prepare noble-metal 
clusters by using $N, N^{\prime}$-dimethylformamide (DMF) that was shown to act as both a mild reducing and a stabilizing agent. Unfortunately these authors synthesized non-luminescent nanoparticles with sizes larger than $5 \mathrm{~nm}$. This is unambiguously obvious from the intense surface plasmon resonance absorption bands appearing in the visible region. Later Liu et al. ${ }^{47}$ extended this idea of using DMF as both a reducing and a stabilizing agent, and they succeeded in developing a surfactant-free method allowing to synthesize highly stable and luminescent Au clusters which were subsequently terminated with various ligands. However, in order to successfully synthesize these clusters using DMF, as a reducing and a stabilizing agent, the reaction mixture needs to be refluxed at a high temperature $\left(153^{\circ} \mathrm{C}\right)$. The high boiling point of DMF makes the purification and drying process quite difficult.

In theory, the use of any solvent with a low boiling point and sufficient reducing ability may replace DMF in such reactions. We found out that acetonitrile is the solvent which matches those criteria and thus can be employed for such reactions. Although the use of acetonitrile failed to synthesise gold clusters, this solvent is very well suited for the preparation of highly stable, luminescent $\mathrm{Ag}$ clusters. Here we report a facile route that was elaborated to synthesize luminescent $\mathrm{Ag}$ nanoclusters with sizes of around 1.3-1.5 nm, stabilized by poly( $N$-vinylpyrrolidone) (PVP, K30, $M_{\mathrm{W}}$ $40 \mathrm{kDa}$ ), where acetonitrile was used as both solvent and a reducing agent. This method needs a low refluxing temperature $\left(75{ }^{\circ} \mathrm{C}\right.$ in contrast to $153{ }^{\circ} \mathrm{C}$ for DMF). The low boiling point of acetonitrile considerably facilitates subsequent processing of the reaction products. The photoluminescence (PL) lifetime of the Ag clusters were found to be in the nanosecond (ns) time region. Furthermore, the ultrafast excited-state dynamics of the Ag clusters was studied by performing femtosecond (fs) transient absorption experiments utilizing $150 \mathrm{fs}$ pump pulses at $387 \mathrm{~nm}$ and white-light probe pulses in the visible region. The excited-state relaxation dynamics at short time scales (femtoseconds to picoseconds) were conceived to occur via ultrafast internal conversion ( $0.3 \mathrm{ps})$ from initially excited, higher lying electronic states to the longer-lived first excited $\left(\mathrm{S}_{1}\right)$ state and by trapping processes due to surface states.

\section{Experimental section}

\subsection{Materials}

Silver nitrate $\left(99.9999 \% \mathrm{AgNO}_{3}\right)$ was obtained from Sigma-Aldrich, poly( $N$-vinylpyrrolidone) K30 ((PVP; $\left.\left.\left(\mathrm{C}_{6} \mathrm{H}_{9} \mathrm{ON}\right)_{n}\right), M_{\mathrm{w}} \sim 40 \mathrm{kDa}\right)$ from Fluka, $\mathrm{NaBH}_{4}(97 \%)$ acetonitrile (LC-MS Chromasolv ${ }^{\circledR} \geq 99.9 \%$ ) from Fluka, $N, N^{\prime}$-dimethylformamide from Roth ( $\left.\geq 99.8 \%\right)$, absolute ethanol and acetone (99.9\%) were from Merck, and Membra-Cel ${ }^{\circledR}$ dialysis tubing (regenerated cellulose, MWCO $7 \mathrm{kDa}$ ) from SERVA. All materials and solvents were used as received from the suppliers without further purification. In all syntheses milli-Q deionised water (18 $\mathrm{M} \Omega$ ) was used.

\subsection{Synthesis of the Ag clusters}

First, $0.075 \mathrm{M}$ of poly( $N$-vinylpyrrolidone) (in terms of monomeric units) was dissolved in $50 \mathrm{ml}$ of acetonitrile in a threenecked round bottom flask fitted with a reflux condenser under an argon atmosphere. The whole system was then placed on a heating plate in a silicon-oil bath and slowly heated, until the temperature reaches $75{ }^{\circ} \mathrm{C}$. An aqueous solution of silver nitrate $(0.5 \mathrm{ml}$ of $0.1 \mathrm{M})$ was then added at once to this pre-heated solution under vigorous stirring. The reaction was allowed to proceed for at least 6 hours under reflux. In the mean time, formation of the clusters can be checked by applying $U V$ radiation $\left(\lambda_{\max } \approx 364 \mathrm{~nm}\right)$ to the reaction mixture, from where a blue luminescence was observed which was getting more and more intense with increasing refluxing time. Instead of acetonitrile, $N, N^{\prime}$-dimethylformamide can also be used as both solvent and a reducing agent. This synthesis recipe yielded green luminescent Ag clusters. From now onwards we will designate the Ag clusters prepared using acetonitrile as A-AgPVP and the one prepared using DMF as D-AgPVP.

\subsection{Purification of the Ag clusters}

After the completion of the reaction, the flask was allowed to cool down to room temperature. The reaction mixture was then centrifuged thrice at $12000 \mathrm{rpm}$ for $30 \mathrm{~min}$ at room temperature $\left(25^{\circ} \mathrm{C}\right)$ to remove excess PVP and the larger nanoparticles. The supernatant obtained from this step was then evaporated to dryness under reduced pressure. The solid thus obtained was washed with an acetone-water mixture (v/v:3/1) and then separated by centrifugation at $1000 \mathrm{rpm}$. This step was repeated several times, in order to remove the residual unbound polymer from the cluster solution. Finally the Ag clusters were dissolved in $3 \mathrm{ml}$ of deionised water and then dialysed overnight using a cellulose ester membrane tube with a cut-off molecular-weight (MWCO) of $7 \mathrm{kDa}$ in $100 \mathrm{ml}$ of water.

\subsection{Synthesis of Ag@PVP nanoparticles}

Ag@PVP nanoparticles were synthesized by mixing $1 \mathrm{mM}$ of $\mathrm{AgNO}_{3}$ to $2 \mathrm{mM}$ of PVP (in terms of monomeric units) in $100 \mathrm{ml}$ of methanol followed by adding freshly prepared $0.2 \mathrm{M}$ of $\mathrm{NaBH}_{4}$ in $20 \mathrm{ml}$ of water under vigorous stirring. After $1 \mathrm{~h}$ stirring, a dark brown precipitate was formed that was separated by centrifugation (8000 rpm for $15 \mathrm{~min}$ ) at room temperature and then washed thrice with a water-methanol (20\%) mixture followed by ethanol. After removing the excess solvent, finally a dark brown powder of the nanoparticle was obtained which shows the characteristic surface plasmon resonance peak at $397 \mathrm{~nm}$ in the absorption spectrum. Though in this contribution the main emphasis will be given on AgPVP clusters, Ag@PVP nanoparticles have been synthesized only to give an insight into the difference in the sizes and optical properties between silver nanoparticles and silver clusters.

The as-prepared nanoparticles and also the clusters are easily soluble in water, methanol, ethanol and other protic solvents. For further characterization an aqueous solution of the nanoparticles and an ethanolic (absolute ethanol) solution of the clusters were used.

\section{Methods}

\subsection{High-resolution transmission electron microscopy (HRTEM)}

The HRTEM images were recorded using a Philips CM 300 UltraTwin microscope. The measurements were carried out at 
an accelerating voltage of $300 \mathrm{kV}$ in the bright-field mode. The TEM images were taken using a Zeiss EM 900 instrument operating at $120 \mathrm{kV}$ accelerating voltage. The samples for HRTEM and TEM were prepared by dropcasting 5 microliters of the ethanolic solution of the $\mathrm{Ag}$ nanoparticles and $\mathrm{Ag}$ clusters onto an ultrathin carbon coated copper grid (300 mesh) followed by drying in a vacuum at $25{ }^{\circ} \mathrm{C}$ for 2 days.

\subsection{X-ray photoelectron spectroscopy}

The chemical composition of the cluster was investigated by X-ray photoelectron spectroscopy (XPS, PHI 5600 XPS spectrometer). Monochromatic $\mathrm{Al} \mathrm{K}_{\alpha}$ was used as the X-ray source.

\subsection{Steady-state optical spectroscopy}

The PL spectra were recorded on a Jobin-Yvon FluoroMax-3 spectrofluorometer using the magic-angle polarization configuration and a slit width of $5 \mathrm{~nm}$ for both excitation and emission spectra. The UV/Vis absorption spectra were recorded on a Perkin Elmer UV/Vis absorption spectrometer lambda 2. All experiments were performed at room temperature using quartz cuvettes with an optical path length of $10 \mathrm{~mm}$.

The PL quantum yields of the Ag clusters were estimated using a fluorescence standard. The following relative comparison method was used to evaluate the quantum yield:

$$
\frac{Q_{\mathrm{t}}}{Q_{\mathrm{s}}}=\frac{\left(I_{\mathrm{t}} / A_{\mathrm{t}}\right) \eta_{\mathrm{t}}^{2}}{\left(I_{\mathrm{s}} / A_{\mathrm{s}}\right) \eta_{\mathrm{s}}{ }^{2}},
$$

where $Q$ is the PL quantum yield, $I$ the integral area under the PL spectrum, $\eta$ the refractive index of the solvent, and $A$ the absorption at the selected excitation wavelength. The subscripts " $t$ " and "s" represent the test sample and fluorescence standard, respectively.

\subsection{Time-resolved optical spectroscopy}

PL decay profiles were recorded using the time-correlated singlephoton counting (TCSPC) PL spectroscopy technique. This time resolved measurement of the PL intensity was carried out on the TCSPC spectrometer Fluorolog-3 (Jobin Yvon) equipped with a microchannel plate (Hamamatsu, R3809U-50) that provides a time resolution of about 60 ps.

Femtosecond transient absorption spectroscopy experiments were conducted using a Clark MXR CPA 2101 laser system in conjunction with an Ultrafast TAPPS HELIOS detection system, consisting primarily of a glass fiber based spectrometer. The output pulses at $387 \mathrm{~nm}$ with a $150 \mathrm{fs}$ pulse and a $1 \mathrm{kHz}$ repetition rate were used as pump pulses. They were obtained by amplifying and frequency doubling the $775 \mathrm{~nm}$ seeding pulses of the $\mathrm{Er}^{3+}$-doped glass fiber oscillator in a regenerative chirped-pulse titanium-sapphire amplifier and with the frequency doubling BBO crystal in the nonlinear optical amplifier (NOPA), respectively. All samples were pumped at excitation densities between $1.14 \times 10^{9}$ and $1.90 \times 10^{9} \mathrm{~W} \mathrm{~cm}^{-2}$.

The samples consisting of $1 \mathrm{mg}$ of Ag clusters dispersed in $0.5 \mathrm{ml}$ of absolute ethanol were irradiated in quartz cuvettes with a thickness of $2 \mathrm{~mm}$. A fraction of the fundamental was simultaneously passed through a sapphire plate $(3 \mathrm{~mm})$ to generate the fs white-light continuum between 400 and $1400 \mathrm{~nm}$. The chirp between 400 and $750 \mathrm{~nm}$ was approximately 350 fs. Transient absorption spectra of the Ag clusters in ethanol were taken at delay times between -0.5 ps and 4 ns. They were recorded in the visible region between 420 and $750 \mathrm{~nm}$.

\section{Results and discussion}

The Ag clusters were synthesized by reducing $\mathrm{Ag}^{+}$ions in acetonitrile at $75{ }^{\circ} \mathrm{C}$ and in DMF at $153{ }^{\circ} \mathrm{C}$. However, to produce chemically stable, luminescent $\mathrm{Ag}$ clusters, the just formed clusters must be stabilized in time by suitable stabilizing agents. As a stabilizing agent, we have used the biocompatible homopolymer poly( $N$-vinylpyrrolidone) (PVP) that contains an amide group in its monomeric unit and is known to have good stabilizing ability for transition metal particles. The successful use of PVP as a stabilizing agent for the synthesis of $\mathrm{Ag}$ and $\mathrm{Au}$ clusters was already demonstrated by various other groups. ${ }^{19,45,46}$ In the presence of PVP, the $\mathrm{Ag}^{+}$ions were chelated. Subsequent reduction of the $\mathrm{Ag}^{+}-\mathrm{PVP}$ complexes by acetonitrile or DMF at the refluxing temperature of the respective solvent provided the formation of PVP-stabilized Ag clusters. Remarkably, the same reduction reaction of $\mathrm{Ag}^{+}-\mathrm{PVP}$ complexes, when performed at temperatures lower than the boiling point of the respective reducing agent and solvent, will yield large-sized $\mathrm{Ag}$ nanoparticles.

X-ray photoelectron spectroscopy (XPS) experiments were performed to examine the oxidation state of Ag in A-AgPVP clusters. The binding-energy values of $368.48 \mathrm{eV}$ and $374.57 \mathrm{eV}$ are assigned to $\mathrm{Ag} 3 \mathrm{~d}_{5 / 2}$ and $\mathrm{Ag} 3 \mathrm{~d}_{3 / 2}$ states, respectively (Fig. 1A). These values are very typical for elemental $\mathrm{Ag}(0)$ and therefore indicate the formation of ultrasmall $\mathrm{Ag}$ clusters. The binding energy of N1s and O1s were found to be $400.28 \mathrm{eV}$ and $533.28 \mathrm{eV}$ (Fig. 1B and C). The much larger binding energy value of the O1s orbital $(533.4 \mathrm{eV})$ in the A-Ag-PVP clusters, when compared to that of the O1s orbital $(531.3 \mathrm{eV})$ for the pure PVP sample, suggests efficient interactions between the oxygen atom of the monomer and surface $\mathrm{Ag}$ atoms. Such interactions between the oxygen atom of the $-\mathrm{C}=\mathrm{O}$ group of $\mathrm{PVP}$ and $\mathrm{Ag}$ atoms were also reported previously by Chen et al. ${ }^{49}$

TEM and HRTEM images of the Ag@PVP nanoparticles are shown in Fig. 2A and B. The Ag@PVP nanoparticles have a singlecrystalline structure and an average size of $6.5 \mathrm{~nm}$ (Fig. 2D). However, because of their ultra-small sizes the Ag clusters could not be detected in TEM. The HRTEM image recorded at an accelerating voltage of $300 \mathrm{kV}$ provides some clue about the formation of very small tiny clusters. An exemplary HRTEM image of the as-synthesized A-AgPVP clusters is depicted in Fig. 2C. The image shows some particles of which most of them have no definite shape. However we were able to resolve few spherical particles in the image which are marked by red circles. The sizes of these spherical particles were estimated in the range of 1.3 to $1.5 \mathrm{~nm}$. The HRTEM image histogram of the size distribution of the Ag@PVP nanoparticles is shown in Fig. 2D.

Fig. 3 depicts the UV/Vis absorption spectra of the $\mathrm{Ag}$ clusters and Ag@PVP nanoparticles. The absorption spectrum 

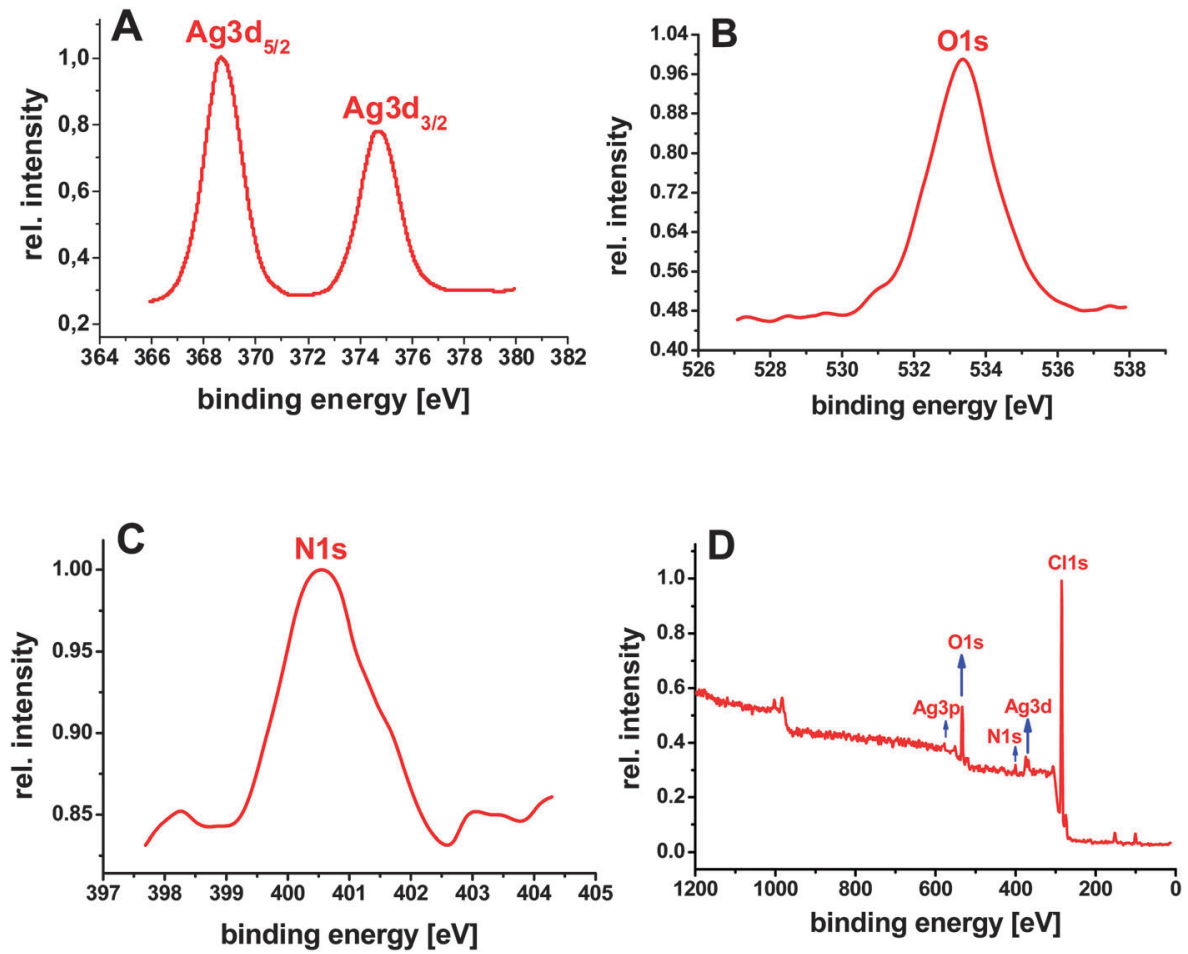

Fig. 1 (A-C) are the XPS spectra of Ag3d, O1s and N1s regions of the A-AgPVP clusters respectively; the survey XPS spectra of the A-AgPVP clusters is depicted in (D).
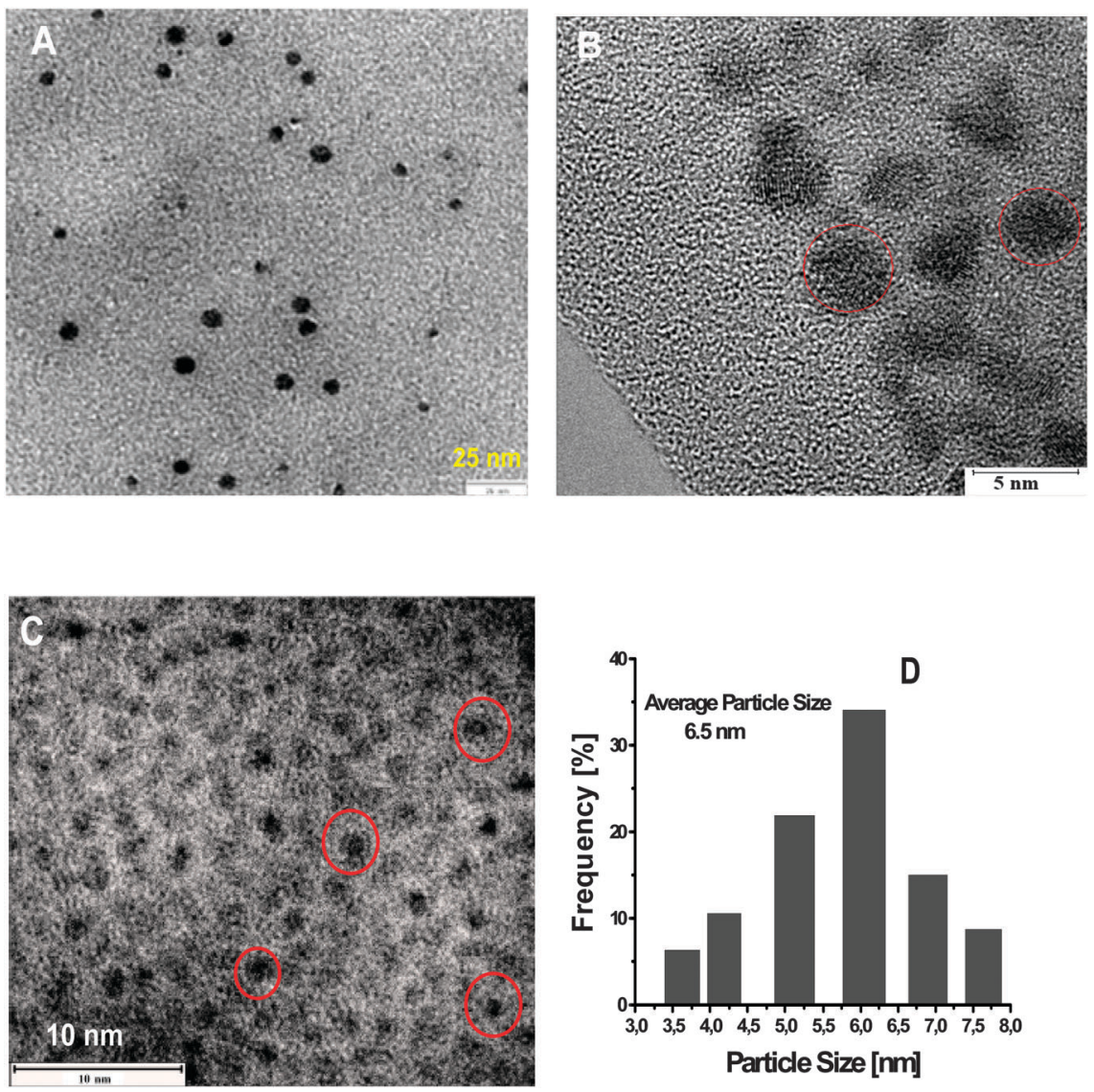

Fig. 2 TEM image of the Ag@PVP nanoparticles (A); HRTEM images of the Ag@PVP nanoparticles (B) and A-AgPVP clusters (C); (D) represents the HRTEM image histogram of the size distribution of the Ag@PVP nanoparticles. 


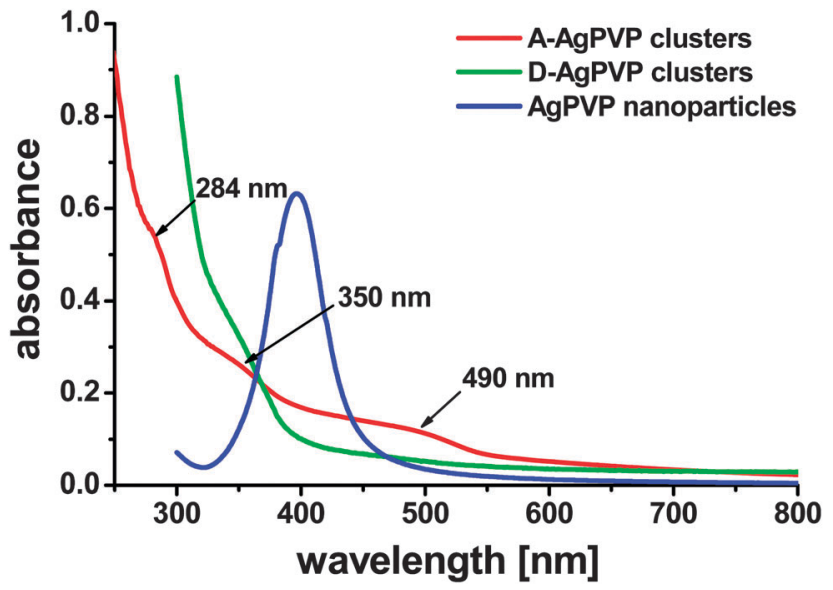

Fig. 3 UV/Vis absorption spectra of the as-synthesized A-AgPVP clusters (red solid line), D-AgPVP clusters (green solid line) and Ag@PVP nanoparticles (blue solid line).

of the Ag@PVP nanoparticles shows the characteristic surface plasmon resonance peak at $397 \mathrm{~nm}$, whereas the absorption spectra of the $\mathrm{Ag}$ clusters do not contain this specific feature but consist of different, significantly structured, broad absorption bands. The A-AgPVP clusters exhibit weakly pronounced absorption peaks at $284 \mathrm{~nm}(4.36 \mathrm{eV}), 350 \mathrm{~nm}(3.54 \mathrm{eV})$, and $490 \mathrm{~nm}(2.53 \mathrm{eV})$, which are superimposed on a broad absorption background. On the other hand, the absorption spectrum of the D-AgPVP clusters contains a shoulder-like absorption feature at around $350 \mathrm{~nm}$. These absorption bands may be assigned to discrete electronic transitions between molecularlike energy states and are therefore characteristic of clusters as essentially consisting of surface atoms. Recently, Zhang et al. ${ }^{34}$ reported the photo-activated synthesis of luminescent Ag clusters $\left(\mathrm{Ag}_{4}-\mathrm{Ag}_{9}\right)$ dispersed in polymer microgel, which show a broad absorption band at 330-350 $\mathrm{nm}$ along with a peak at $490 \mathrm{~nm}$ in the UV/Vis absorption spectrum. Water soluble, luminescent

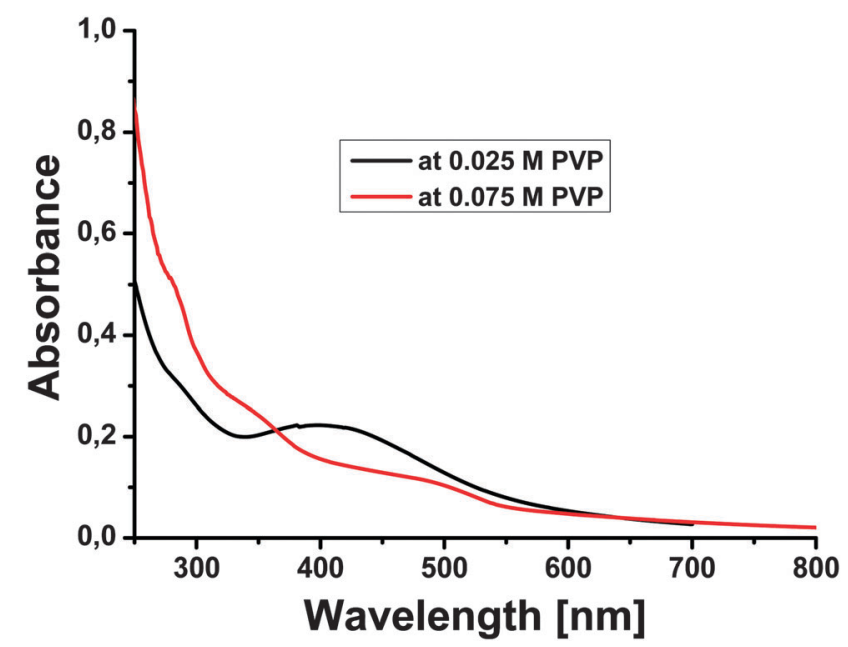

Fig. 4 UV/Vis absorption spectra recorded after $5 \mathrm{~h}$ of reaction between $\mathrm{AgNO}_{3}$ and PVP solution in acetonitrile at reflux. The PVP concentrations used are $0.075 \mathrm{M}$ (red solid line) and 0.025 M (black solid line).

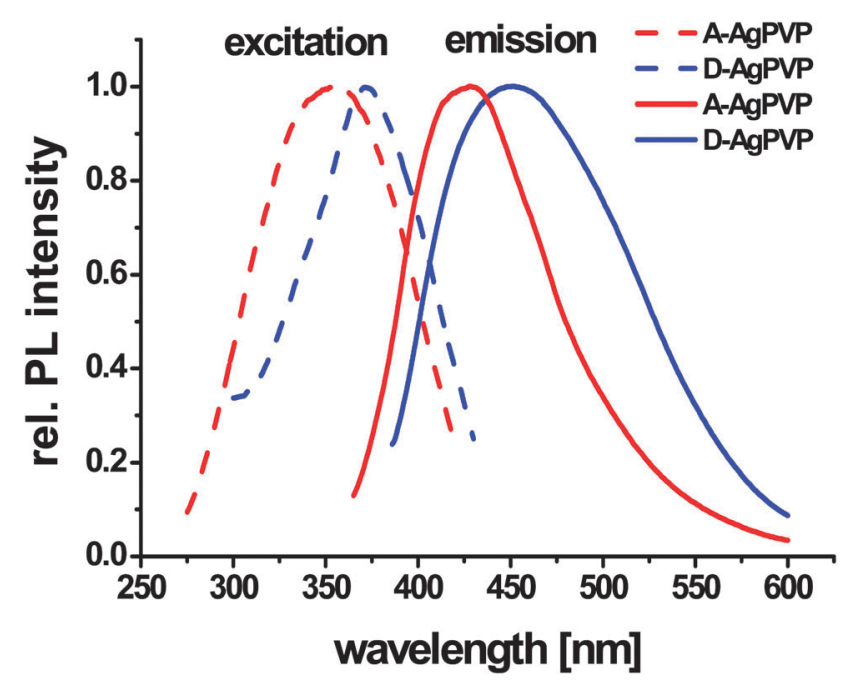

Fig. 5 PL excitation spectra of the A-AgPVP clusters (red dashed line) and D-AgPVP clusters (blue dashed line) and PL emission spectra of the A-AgPVP clusters (red solid line) and D-AgPVP clusters (blue solid line).

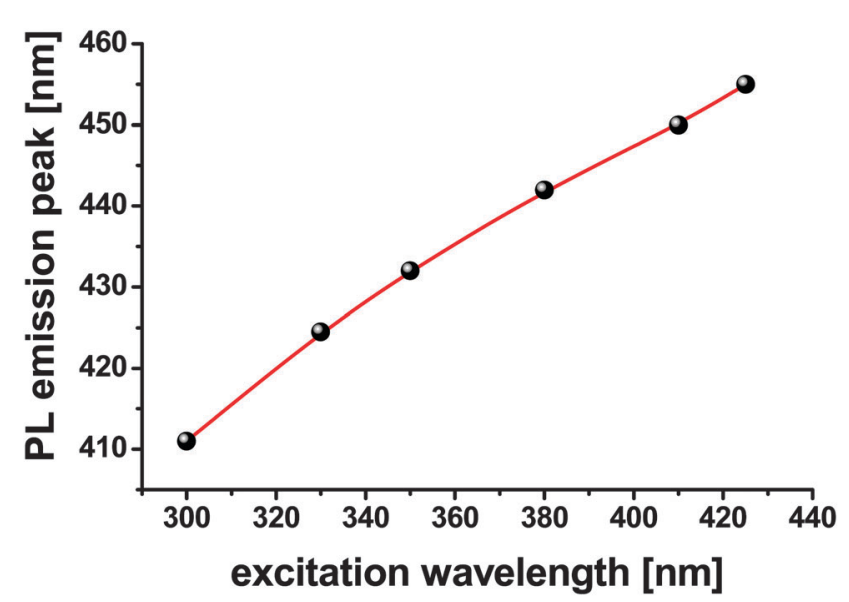

Fig. 6 The dependence of the PL emission peaks of the A-AgPVP clusters on the excitation wavelength.

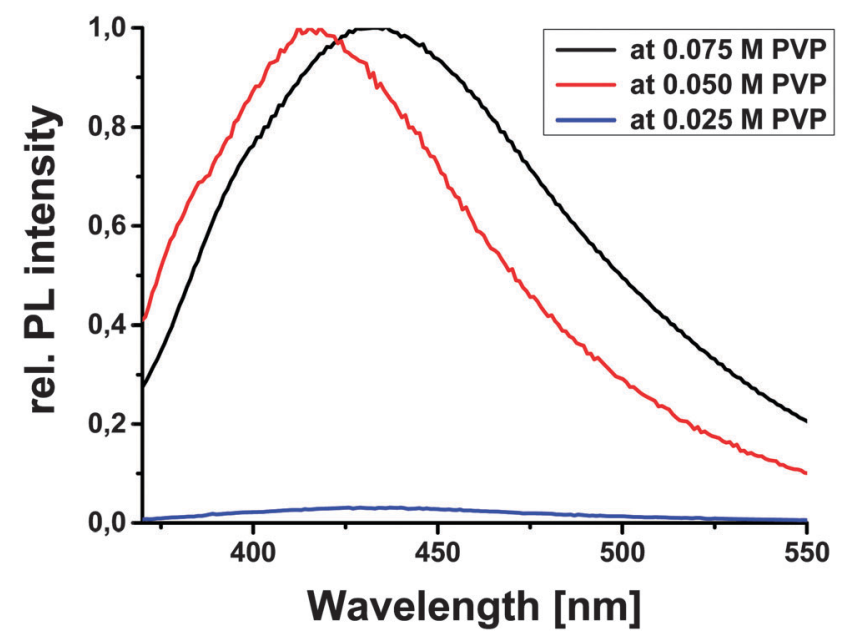

Fig. $7 \mathrm{PL}$ emission spectra of the A-AgPVP clusters synthesized using various concentrations of PVP; $0.075 \mathrm{M}$ (black solid line), $0.050 \mathrm{M}$ (red solid line) and $0.025 \mathrm{M}$ (blue solid line) respectively. 


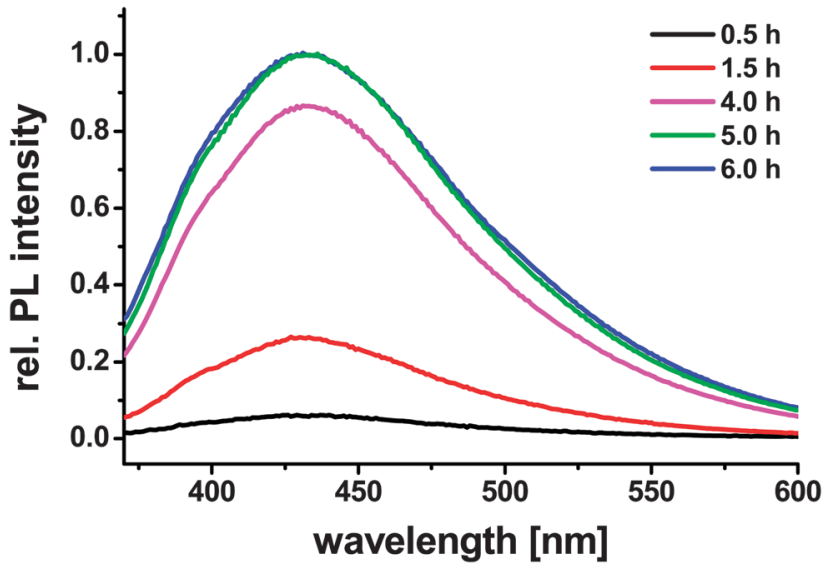

Fig. $8 \mathrm{PL}$ emission spectra of the as-synthesized A-AgPVP clusters excited at $350 \mathrm{~nm}$ and recorded at different reaction times.

Ag clusters stabilized by dihydrolipoic acid (DHLA) were observed to exhibit similar absorption features. ${ }^{17}$ Several other research groups synthesized Ag clusters with various sizes using different capping agents, and for all clusters they found very similar absorption features in the UV and visible region of the spectrum. ${ }^{35,36,42,43,48}$ In agreement with these results, the particular structural features of the absorption spectra may be associated with $\mathrm{Ag}$ clusters that consist of 5 to 9 atoms. Moreover, the UV/Vis absorption spectra of the as-synthesized $\mathrm{Ag}$ clusters did not change over a one-month storage time which proves their great stability.
The PVP concentrations play an important role in the formation of Ag clusters. When 0.075 M PVP (in terms of monomer units) is used in the reaction, luminescent $\mathrm{Ag}$ clusters stabilized by the PVP polymer were obtained. However, large-sized nonluminescent $\mathrm{Ag}$ nanoparticles were produced when a small concentration of $0.025 \mathrm{M}$ PVP was used in the reaction (Fig. 4). Here in this reaction PVP acts as a cross-linking agent for agglomeration of clusters. At high concentrations, the coil-like polymer cages encapsulated the clusters better and protected the clusters from the quencher in solution. Moreover, a larger concentration of the stabilizer led to a smaller growth rate of the particles, and in turn facilitated the formation of clusters instead of nanoparticles. At low stabilizer concentrations, the reduction took place so fast that the polymer could not complex the silver ions in an efficient manner, which resulted in particle aggregation and consequently, large-sized nanoparticles were formed. Fig. 4 shows the UV/Vis absorption spectra recorded after $5 \mathrm{~h}$ of the reaction between $\mathrm{AgNO}_{3}$ and PVP in acetonitrile at reflux. For low PVP concentrations the formation of $\mathrm{Ag}$ nanoparticles is evident from the appearance of the surface plasmon resonance band at $405 \mathrm{~nm}$ in the UV/Vis absorption spectra.

Upon photoexcitation at $364 \mathrm{~nm}$ the A-AgPVP clusters emit a blue photoluminescence (PL) emission peak at $424 \mathrm{~nm}(2.93 \mathrm{eV})$, while the PL emission spectrum of the D-AgPVP clusters is shifted to the spectral range at around $450 \mathrm{~nm}(2.73 \mathrm{eV})$ (Fig. 5). The corresponding PL excitation spectra exhibit their respective maximum at $350 \mathrm{~nm}(3.54 \mathrm{eV})$ for the A-AgPVP clusters and at $370 \mathrm{~nm}(3.35 \mathrm{eV})$ for the D-AgPVP clusters. The narrow PL
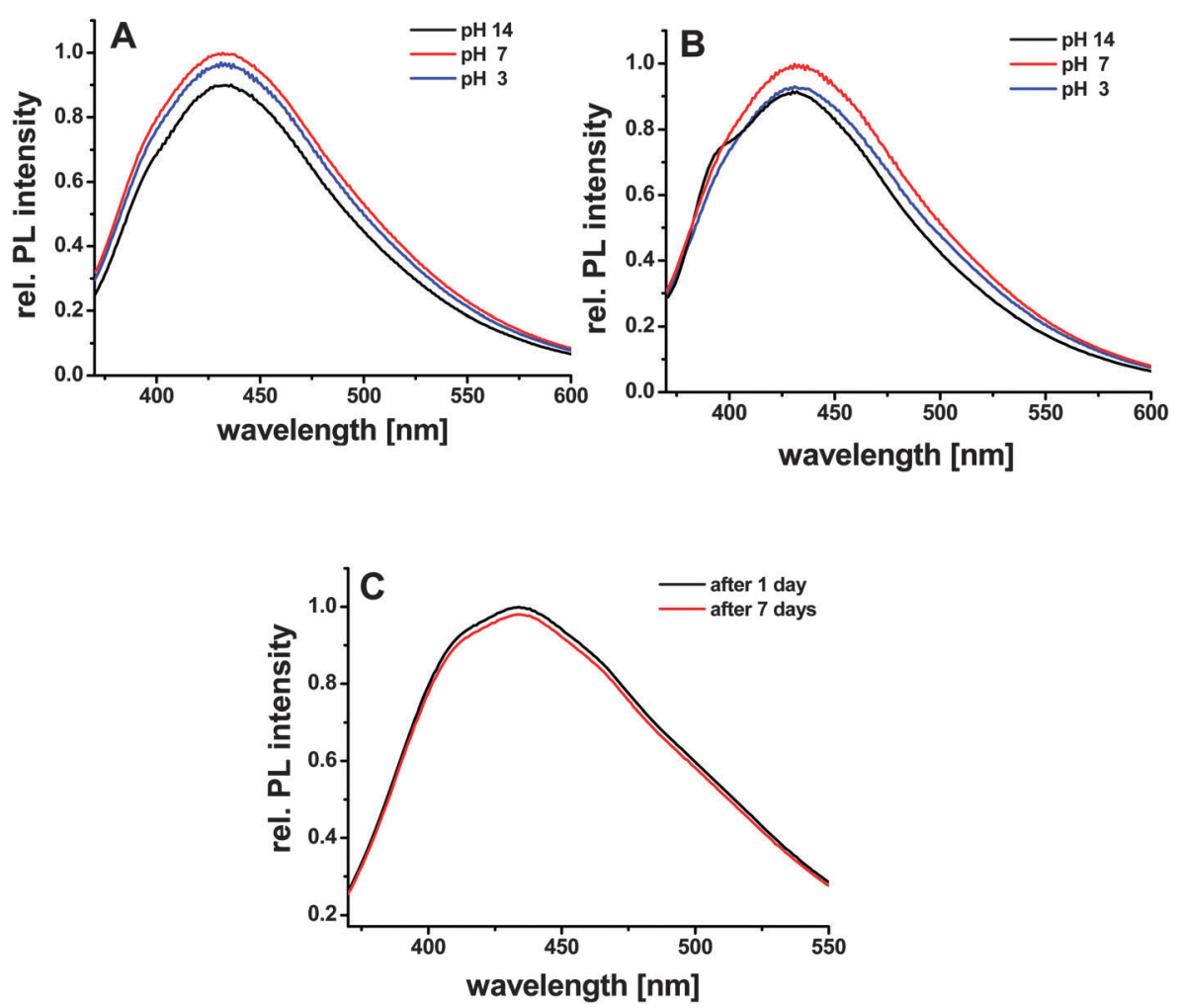

Fig. $9 \mathrm{PL}$ emission spectra of A-AgPVP clusters in an aqueous solution at $\mathrm{pH}$ values of 3, 7 and 14 after 7 days (A) and 14 days (B) of preparation; PL emission spectra of the A-AgPVP clusters in a $0.5 \mathrm{M} \mathrm{NaCl}$ aqueous solution after 7 and 14 days of preparation (C). 
emission profile indicates a small size distribution of the $\mathrm{Ag}$ clusters. The narrow size distribution is also evident from the PL emission spectrum of the A-AgPVP clusters, which was excited at different wavelengths ranging from $320 \mathrm{~nm}$ to $430 \mathrm{~nm}$ (Fig. 6). Such a shift of the PL emission peak to longer wavelengths with increasing excitation wavelengths indicates the presence of $\mathrm{Ag}$ clusters with different sizes. The linear dependence of the wavelength of the PL emission maximum on the PL excitation wavelength was already reported elsewhere ${ }^{31,42,50}$ and is tentatively assigned to inter-band transitions from the sub-merged and quasi continuum $5 \mathrm{~d}$ band to the lowest un-occupied conduction band states of Ag.

The PL emission maxima were observed to change relative to PVP concentration. Fig. 7 shows the PL emission spectra of the A-AgPVP clusters prepared using different concentrations of PVP $(0.075 \mathrm{M}, 0.05 \mathrm{M}$, and $0.025 \mathrm{M}$, respectively). Clearly no luminescent Ag clusters were formed for a PVP concentration of $0.025 \mathrm{M}$. On the other hand, a slight red shift of the PL emission maximum was observed, when a threefold larger PVP concentration (0.075 M PVP) was used. Obviously PVP provides a cross-linking environment for the clusters so that the degree of this cross linking depends on the PVP concentration. Different concentrations of PVP impart different chemical and electronic environments for the clusters, which results in different PL emission energies.

It has to be mentioned that neither the pure PVP solution nor solutions containing PVP with adsorbed or embedded Ag ions were found to emit detectable fluorescence under the same photo-excitation conditions. This unambiguously demonstrates that the observed PL has to be associated with the formation of reduced silver clusters embedded in a PVP matrix. Fig. 8 depicts the reaction-time evolution of the PL emission spectra of A-AgPVP clusters. The reaction time of the A-AgPVP clusters corresponds to the duration of their synthesis when the reaction mixture was heated under reflux conditions. The PL emission was excited at $350 \mathrm{~nm}$. Obviously, the PL intensity of $\mathrm{Ag}$ clusters increases upon increasing the reaction time and reaches its maximum value for clusters synthesized over 5 hour refluxing. This implies that the reaction has come to an end after the 5 hour refluxing time.

The PL quantum yields of the A-AgPVP and D-AgPVP clusters were determined using quinine sulphate as the fluorescence standard, and the obtained values are $\Phi_{\mathrm{A}-\mathrm{AgPVP}}=3.5 \%$ and $\Phi_{\text {D-AgPVP }}=2.7 \%$ respectively. Although reports on red-emitting $\mathrm{Ag}$ clusters are frequent, only few were published on blueemitting Ag clusters. Recently, González et al. ${ }^{44}$ developed an electrochemical synthesis procedure to synthesize blue-emitting and $\mathrm{Ag}_{6}$ clusters that were stabilized with dodecanethiol and tetrabutyl ammonium. The $\mathrm{Ag}_{6}$ clusters exhibit PL emission at around $420 \mathrm{~nm}$ with a quantum yield of $1.2 \%$. The PL emission spectrum of these $\mathrm{Ag}$ clusters nicely coincides with that of the here synthesized A-AgPVP clusters, whereas the PL quantum yield is significantly smaller than that of the A-AgPVP clusters with a value of $3.5 \%$. Furthermore, Ag clusters stabilized with the polyethyleneimine polymer were reported to emit PL in the range of $420-490 \mathrm{~nm}^{.44,51,52}$
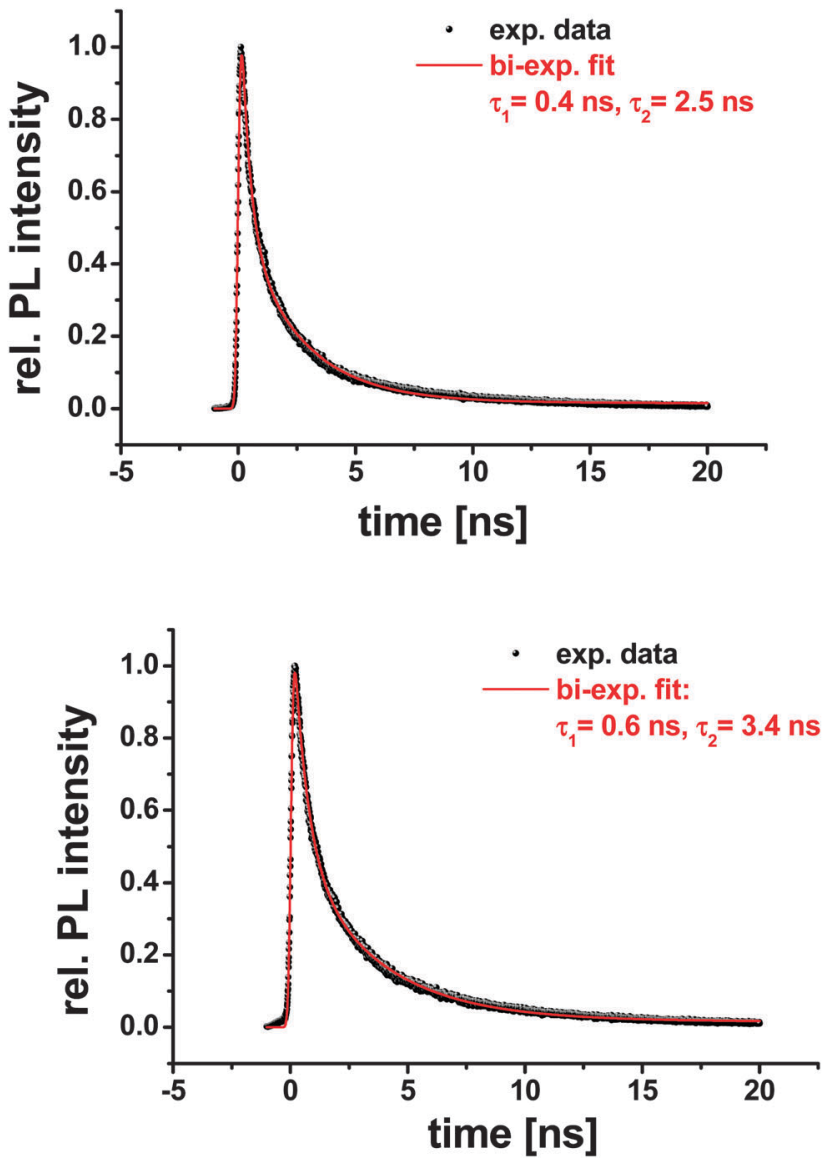

Fig. 10 PL emission decays of the A-AgPVP clusters (at top) and the D-AgPVP clusters (at bottom), both excited at $403 \mathrm{~nm}$.

The A-AgPVP clusters show great chemical stability towards various kinds of solvents. To examine the colloidal stability of these clusters, their PL emission spectrum was measured in aqueous solution at different $\mathrm{pH}$ values and in an aqueous solution with a high ionic strength $(0.5 \mathrm{M} \mathrm{NaCl})$. Almost the same PL emission intensity of the A-AgPVP clusters was observed under all solvent conditions and even after 14 days of preparation (Fig. 9A-C). These results suggest the high chemical stability of the A-AgPVP clusters in acidic and basic solvents as well as in solution with high ionic strengths. Strikingly, the addition of $\mathrm{NaCl}$ to the solvent is associated with a small red shift of the PL emission maximum of the A-AgPVP clusters. Moreover, the addition of a reducing agent such as $\mathrm{NaBH}_{4}$ or of an oxidizing agent like $\mathrm{H}_{2} \mathrm{O}_{2}$ to the A-AgPVP cluster colloid was not observed to alter the spectral features of their PL emission and UV/Vis absorption spectra. These experimental results prove both the high stability of the $\mathrm{Ag}$ clusters and the absence of any $\mathrm{Ag}^{+}$ions in the solution.

PL lifetime measurements of the A-AgPVP and D-AgPVP clusters were performed by detecting their PL emission at $430 \mathrm{~nm}$ and $450 \mathrm{~nm}$, respectively. The PL emission decay curves were simulated employing a convolution of the apparatus response with a bi-exponential decay function $f(t)$ being $f(t)=a_{1} \times \exp \left(-t / \tau_{1}\right)+$ $a_{2} \times \exp \left(-t / \tau_{2}\right)$ (Fig. 10). For the A-AgPVP clusters, the predominant slow decay component, with $a_{2}=0.7$ and $\tau_{2}=2.5 \mathrm{~ns}$, is assigned 

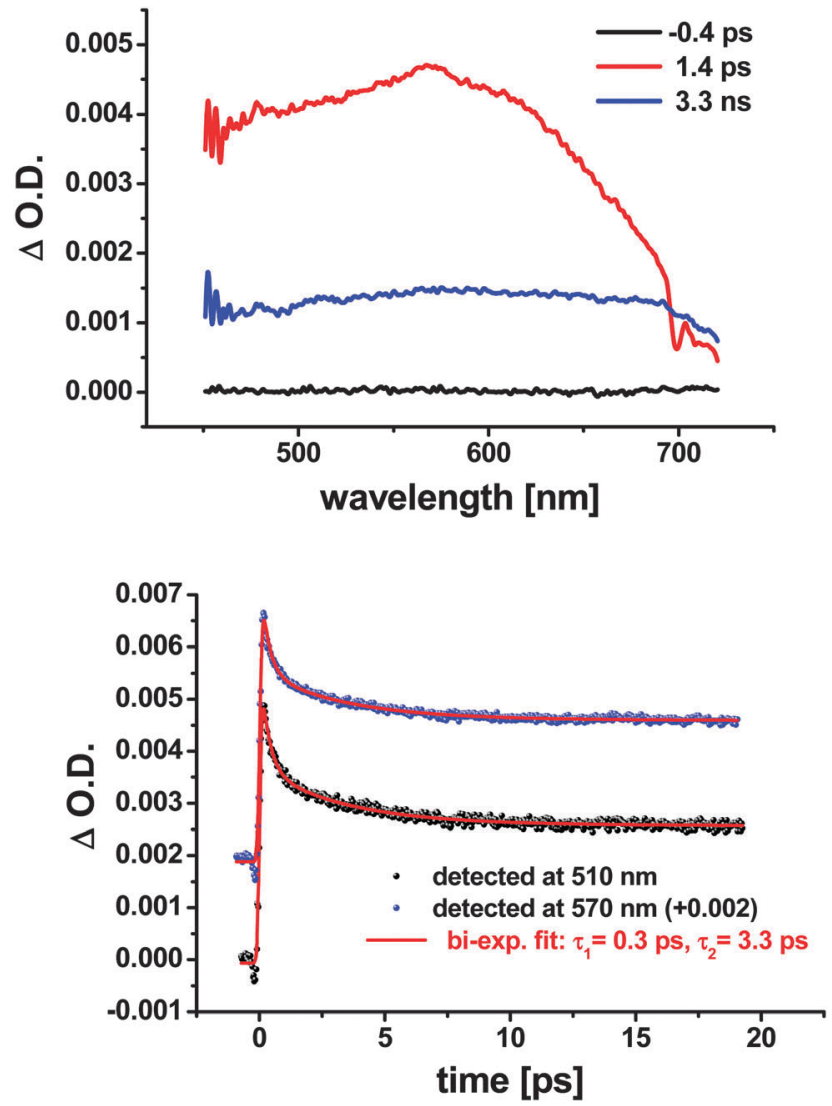

Fig. 11 Time evolution of the transient absorption spectra (at top) with their characteristic kinetic traces (at bottom) of the A-AgPVP clusters.

to the PL emission decay of $\mathrm{Ag}_{6}$ clusters, whereas the larger time constant $\left(\tau_{2}=3.4 \mathrm{~ns}, a_{2}=0.6\right)$ of the D-AgPVP clusters is assigned to the lifetime of the emitting state of $\mathrm{Ag}_{7}$ clusters. The respective faster PL emission decay component of the A-AgPVP and D-AgPVP clusters ( $\tau_{1}=0.4 \mathrm{~ns}$ or $\left.0.6 \mathrm{~ns}\right)$ might reveal the contribution to the PL emission due to smaller Ag clusters.

Complementary information of ultrafast excited-state relaxation dynamics of A-AgPVP clusters was obtained from fs transient absorption spectroscopy experiments. Fig. 11 presents the time evolution of the transient absorption spectra of the excited-state relaxation dynamics of the $\mathrm{Ag}$ clusters following excitation at $387 \mathrm{~nm}$. The spectra exhibit a broad featureless photo-induced absorption signature between 450 and $700 \mathrm{~nm}$ with a maximum at around $570 \mathrm{~nm}$. The short-time scale kinetic traces detected at 510 and $570 \mathrm{~nm}$ display the temporal decay behavior of the overall transient absorption spectrum. The kinetic traces were fit using a bi-exponential function that is deconvolved with a 240 fs Gaussian representing the apparatus response. The bi-exponential decay dynamics comprises a subpicosecond (65\%) and a picosecond decay component $(35 \%)$. The ultrashort lifetime with $\tau_{1}=0.3 \mathrm{ps}$ is attributed to internal conversion as the process that provides ultrafast relaxation dynamics of initially photo-excited, higher electronic states to the $\mathrm{S}_{1}$ state. On the other hand, the larger time constant with $\tau_{2}=3.3 \mathrm{ps}$ is associated with surface trapping of the initially generated excitons.

\section{Conclusions}

In summary, a novel synthesis route was developed which was successfully used to prepare highly luminescent, stable and nearly mono-disperse Ag clusters. Our synthesis recipe consists of a facile, environmentally friendly on-step reaction, in which PVP acts as the stabilizing agent and acetonitrile or DMF serves as both solvent and a reducing agent. The XPS results confirm the complete reduction of silver ions and furthermore verify efficient interactions between the oxygen atom of the monomer unit and the Ag cluster. The PL lifetimes of the A-AgPVP clusters and D-AgPVP clusters were determined to be $2.5 \mathrm{~ns}$ and $3.4 \mathrm{~ns}$, respectively. For the A-AgPVP clusters, fs transient absorption spectroscopy experiments allowed for the observation of bi-exponential ultrafast excited-state relaxation dynamics. The two obtained time constants with $0.3 \mathrm{ps}$ and $3.3 \mathrm{ps}$ were assigned to ultrafast internal conversion emerging from initially higherlying energy states to the emitting $S_{1}$ state and surface capturing of the photo-generated charge carriers. In accord with the actual literature, our evaluated spectroscopic and microscopic results suggest that this synthesis technique predominantly produces ultra-small fluorescent silver clusters having less than 10 atoms in the core.

\section{Acknowledgements}

Support of the Deutsche Forschungsgemeinschaft (graduate school $1161 / 2$ ) is gratefully acknowledged.

\section{References}

1 M. Haruta, Chem. Rev., 2003, 3, 75.

2 L. Li, Q. Guo, J. Li, W. Yan, C. Leng, H. Tang, Q. Lu and B. Tan, J. Mater. Chem. B, 2013, 1, 3999.

3 J. Zheng, P. R. Nicovich and R. M. Dickson, Annu. Rev. Phys. Chem., 2007, 58, 409.

4 B. H. Kim, M. J. Hackett, J. Park and T. Hyeon, Chem. Mater., 2014, 26, 59.

5 S. Parween, A. Ali and V. S. Chauhan, ACS Appl. Mater. Interfaces, 2013, 5, 6484.

6 R. A. Sperling, P. Rivera Gil, F. Zhang, M. Zanella and W. J. Parak, Chem. Soc. Rev., 2008, 37, 1896.

7 X. Wu, X. He, K. Wang, C. Xie, B. Zhou and Z. Qing, Nanoscale, 2010, 2, 2244.

8 S. K. Rastogi, V. J. Rutledge, C. Gibson, D. A. Newcombe, J. R. Branen and A. L. Branen, Nanomedicine, 2011, 7, 305.

9 T. H. Lee, J. I. Gonzalez, J. Zheng and R. M. Dickson, Acc. Chem. Res., 2005, 38, 534.

10 T. G. Schaaff and R. L. Whetten, J. Phys. Chem. B, 2000, 104, 2630.

11 C. B. Murray, D. J. Norris and M. G. Bawendi, J. Am. Chem. Soc., 1993, 115, 8706.

12 W. C. W. Chan and S. Nie, Science, 1998, 281, 2016.

13 Y. G. Sun and Y. Xia, Science, 2002, 298, 2176.

14 T. Zhou, M. Rong, Z. Cai, C. James Yang and X. Chen, Nanoscale, 2012, 4, 4103. 
15 M. C. Pau, C. K. Lo, X. Yang and M. M. F. Choi, J. Phys. Chem. C, 2010, 114, 15995.

16 J. Sun, J. Zhang and Y. Jin, J. Mater. Chem. C, 2013, 1, 138. 17 B. Adhikari and A. Banerjee, Chem. Mater., 2010, 22, 4364.

18 C. A. J. Lin, T. Y. Yang, C. H. Lee, S. H. Huang, R. A. Sperling, M. Zanella, J. K. Li, J. L. Shen, H. H. Wang, H. I. Yeh, W. J. Parak and W. H. Chang, ACS Nano, 2009, 3, 395.

19 H. Tsunoyama, N. Ichikuni, H. Sakurai and T. Tsukuda, J. Am. Chem. Soc., 2009, 131, 70.

20 J. Zheng and R. M. Dickson, J. Am. Chem. Soc., 2002, 124, 13982.

21 J. Sharma, R. C. Rocha, M. L. Phipps, H.-C. Yeh, K. A. Balatsky, D. M. Vu, A. P. Shreve, J. H. Werner and J. S. Martinez, Nanoscale, 2012, 4, 4107.

22 N. Makarava, A. Parfenov and I. V. Baskakov, Biophys. J., 2005, 89, 572 .

23 M. Scolari, A. Mews, N. Fu, A. Myalitsin, T. Assmus, K. Balasubramanian, M. Burghard and K. Kern, J. Phys. Chem. C, 2008, 112, 391.

24 Z. Shen, H. W. Duan and H. Frey, Adv. Mater., 2007, 19, 349.

25 F. Qu, N. Bing Li and H. Qun Luo, J. Phys. Chem. C, 2013, 117, 3548.

26 C. Guo and J. Irudayaraj, Anal. Chem., 2011, 83, 2883.

27 J. Xie, Y. Zheng and J. Y. Ying, J. Am. Chem. Soc., 2009, 131, 888.

28 J. T. Petty, J. Zheng, N. V. Hud and R. M. Dickson, J. Am. Chem. Soc., 2004, 126, 5207.

29 W. W. Guo, J. P. Yuan and E. K. Wang, Chem. Commun., 2009, 3395.

30 C. I. Richards, S. Choi, J. C. Hsiang, Y. Antoku, T. Vosch, A. Bongiorno, Y. L. Tzeng and R. M. Dickson, J. Am. Chem. Soc., 2008, 130, 5038.

31 L. Shang and S. J. Dong, Chem. Commun., 2008, 1088.

32 J. Zheng and R. M. Dickson, J. Am. Chem. Soc., 2002, 124, 13982.

33 J. Zheng, J. T. Petty and R. M. Dickson, J. Am. Chem. Soc., 2003, 125, 7780 .
34 J. G. Zhang, S. Q. Xu and E. Kumacheva, Adv. Mater., 2005, 17, 2336.

35 K. V. Mrudula, T. Udaya Bhaskara Rao and T. Pradeep, J. Mater. Chem., 2009, 19, 4335.

36 M. Farrag, M. Thämer, M. Tschurl, T. Bürgi and U. Heiz, J. Phys. Chem. C, 2012, 116, 8034.

37 L. A. Peyser, A. E. Vinson, A. P. Bartko and R. M. Dickson, Science, 2001, 291, 103.

38 J. Sharma, H.-C. Yeh, H. Yoo, J. H. Werner and J. S. Martinez, Chem. Commun., 2011, 47, 2294.

39 L. Shang, R. M. Dörlich, S. Brandholt, R. Schneider, V. Trouillet, M. Bruns, D. Gerthsen and G. U. Nienhaus, Nanoscale, 2011, 3, 2009.

40 S. Huang, C. Pfeiffer, J. Hollmann, S. Friede, J. Jin-Ching Chen, A. Beyer, B. Haas, K. Volz, W. Heimbrodt, J. M. Montenegro Martos, W. Chang and W. J. Parak, Langmuir, 2012, 28, 8915.

41 A. Henglein, J. Phys. Chem., 1993, 97, 5457.

42 S. Liu, F. Lu and J.-J. Zhu, Chem. Commun., 2011, 47, 2661. 43 H. Xu and K. S. Suslick, ACS Nano, 2010, 4, 3209.

44 B. S. González, M. C. Blanco and M. Arturo López-Quintela, Nanoscale, 2012, 4, 7632.

45 I. Pastoriza-Santos and L. M. Liz-Marzán, Langmuir, 1999, 15, 948.

46 I. Pastoriza-Santos and L. M. Liz-Marzán, Langmuir, 2002, 18, 2888.

47 X. Liu, C. Li, J. Xu, J. Lu, M. Zhu, Y. Guo, S. Cui, H. Liu, S. Wang and Y. Li, J. Phys. Chem. C, 2008, 112, 10778.

48 N. Cathcart and V. Kitaev, J. Phys. Chem. C, 2010, 114, 16010. 49 B. Yin, H. Ma, S. Wang and S. Chen, J. Phys. Chem. B, 2003, 107, 8898.

50 I. Diez, M. I. Kanyuk, A. P. Demchenko, A. Walther, H. Jiang, O. Ikkala and R. H. A. Ras, Nanoscale, 2012, 4, 4434.

51 F. Qu, L. L. Dou, N. B. Li and H. Q. Luo, J. Mater. Chem. C, 2013, 1, 4008.

52 N. Y. Chen, H. F. Li, Z. F. Gao, F. Qu, N. B. Li and H. Q. Luo, Sens. Actuators, B, 2014, 193, 730. 